\title{
Intellectual Development of Primary School-Aged Bilingual Learners
}

\section{Mira Bechmizovna Bogus}

\author{
Federal State Budget Institution of Higher Professional Education «Adyghe State University» (ASU), 385000, Maikop, street \\ Pervomayskaya, 208, Russian Federation; Email: nisadgu@yandex.ru
}

Doi:10.5901/mjss.2015.v6n5p548

\begin{abstract}
The purpose of this work is to determine the effective way to the intellectual development of primary school-aged bilingual learners. The author has made a suggestion about the possibility to increase the intellectual capacity of pupils by means of resource of the existing bilingualism. According to this hypothesis, we have developed a system of intellectual tasks for bilingual learners in both of their languages: mother tongue (Adyghe) and non-mother tongue (Russian). The bilingual intellectual development system includes materials from all school subjects and allows going through all stages of development. In the educational process, the system is implemented in the form of fragments of studies. Intelligent training based on bilingual system material is carried out from the first grade to the fourth one. The performed experimental research brings us to the conclusion that the developed bilingual intellectual development system facilitates the development of the mental abilities of primary school-aged bilingual learners.
\end{abstract}

Keywords: intelligence, development, bilingualism, system, language, primary school pupil.

\section{Introduction}

The most important indicator of the socio-economic well-being of the society is the state of intellectual development of the younger generation, as the children constitute an important reserve of the country, which will determine the level of its economic and spiritual development, the state of science and culture. The capacity of an individual to productive and creative activities depends on the level of his intellectual development (Bogoyavlenskiy, 1992; Vygotskiy, 1982; Davydov, 1986, Zack, 2004; Kalmykova 1981; Matyushkin 2008; Menchinskaya, 1989; Sukhomlinskiy, 1983). Accordingly, the requirement of the XXI century for the school, its graduates, and the educational process is fundamentally changing the main strategic guidelines of the school. The modern school, actively introducing innovative programs and implementing the concept of developing education, has a priority goal: the development of intelligence, creativity and culture of thinking of learners in the context of the general education.

The most important personal characteristic of the learner is his identity with native speakers who speak certain language(s) reflecting the cultural differences, the basic principles of life, the unique linguistic picture of the world (Avrorin 1975; Vereshchagin, 1969; Diachkov, 1991; Zhol 1990; Melnikov, 2003; Mikhailov, 1989). In this regard, the existence of different languages in the personality framework of a learner and the existing bilingualism resource represent a meaningful basis for determination of the entire system of the intellectual development of bilingual learners.

Bilingualism as a social phenomenon is assuming a greater significance and becoming an important attribute of modern society. The twenty-first century has been declared by UNESCO as an era of multilingualism. The opinion of the Council of Europe, which proclaimed multilingualism, multiculturalism, cultural conformity and anthropocentricity as important principles of the national education system, has found a positive response in the Russian education, followed by the actualization of the study of general development of the bilingual learner's personality and the development of his intellectual sphere in particular.

The study of psychological and educational literature reveals that it is a primary school age which is characterized by intensive formation of cognitive processes, positive motivation for training and speech activity, easiness and durability of information memorizing (Vergeles, 1990, Davydov, 1982, Repkin, 1976; Elkonin, 1961, et al.). Unfortunately, due to the lack of theoretical and action-oriented research works, the sensitive period of intellectual development of bilingual pupils of primary school age is not fully realized in the educational process.

Thus, the need to study the problem of intellectual development of primary school-aged bilingual learners is dictated by the demands of modern society, personality and educational process. 


\section{Method}

\subsection{Bilingualism in the intellectual development of learners.}

In the development of a bilingual learner, one of the key positions is the presence of two languages in the cognitive field. Knowledge of language (mother and non-mother tongues), each of which is a reflection of the culture, worldview and attitudes of different ethnic groups; experience of mental activity on the basis of language structures of learners; experience of emotional and value-conscious relation to society, themselves, knowledge and skills, set conditions for the development of mental abilities of learners; complex of knowledge, skills, reflecting the level of intellectual development as a bilingualism resource needs to be implemented in the educational process (Bogus, 2008).

The study is based on the assumption that the implementation of the resource existing in bilingual learners will contribute to their intellectual development.

The essence of the bilingualism resource is revealed through its objective and subjective parts. The objective part of the bilingualism resource consists in the presence of a double sign-symbolic systems, it means the two ways of thought forming. The subjective part of the bilingualism resource is disclosed in characteristics of bilinguals. The individual has (with two developed sign-symbolic systems) the following abilities (to a greater extent compared to a monolingual): a creative approach to the handling of information of different levels, including sensitivity of the bilingual to the semantic relationships between words; ability to generalize the conclusions in two languages; ability to perform the tasks related to the verbal transformation and replacement; developed linguistic memory; linguistic competence.

According to this hypothesis, we have developed a bilingual system of intellectual development, which is based on the idea of a dominant factor - activity. In accordance with this, the execution of tasks and activation of mental abilities have led to the effective intellectual development of bilinguals.

Bilingual system for I-IV grades is a set of subsystems focused on the development of learners' intellectual abilities of different kinds. The system consists of four subsystems: 1) intellectual development at mathematics lessons; 2) intellectual development at natural history lessons; 3) intellectual development at Russian lessons; 4) intellectual development at Adyghe lessons.

In order to achieve a certain stage of development, there is an appropriate set of tasks in each subsystem.

Systems are built on the principle of a different level of complexity. They are based on: increased speed of training problem solving; accelerated transfer of mental operations in the internal plan of action of learners. During the system development, a set of special conditions has been elaborated in order to develop the speech activity of learners which leads to development of figurative, logical and version thinking that as a result increases the speed of learning.

The system has an integrative nature, bringing together the knowledge of the Russian language, Adyghe language, mathematics, reading and outside world, which helps the learner to intensify his mental activity deeply and in a diversified manner.

A differential characteristic of bilingual system tasks is their wide and varied interdisciplinary relation. Exercises are composed with regard to child's inherent interest in different subjects.

The main principles of the distribution of material in the bilingual system tasks are:

1) relationship and interdependence of tasks;

2) gradual complication of tasks;

3) increase in volume of material;

4) increase in speed of task execution;

5) alternation of different activities.

Tasks are composed in accordance with certain stages of intellect development. Feature of the exercise system is that it creates conditions for step-by-step execution of mental actions.

In the process of creation of a system of intellectual development a close attention is paid to the provision of the following conditions ensuring the sustained attention of children: characteristics of the material, degree of difficulty of the material, availability, subject's attitude towards it, and individual characteristics of a person.

\subsection{Type of bilingualism as a basis of the implementation of a bilingual system.}

The research is focused on learners, bilingualism of which has been formed by fundamental way (second language was learned in early childhood, in parallel with the first one), because only a perfect knowledge of the two languages from childhood allows at the primary school age affecting the level of intellectual development by means of language.

Developed bilingual system of intellectual development of bilingual learners is applicable in the implementation of 
the following models of learning: 1) foreign language-based education, both languages are learned as school subjects, 2) mother tongue-based education, mother and non-mother tongues are learned taught as school subjects.

Implemented type of bilingualism is determined as:

- effective (by the comprehension level) (Vereshchagin, 1969) marked by the ability to understand and reproduce the speech production, belonging to the secondary language system;

- two-in-one (by the form of functioning) (Mikhailov, 1989) marked by the comprehension of spoken and written language;

- coordinative at the beginning and mixed afterwards (by the ratio of the two speech mechanisms) (Osgood, 1980), that is, at the beginning the correlated words are interpreted as two separate signs, each of which has its signifier and signified, then the correlated words represent a complex sign consisting of two signifiers and one signified, in this case the distinction between signified is ignored;

- simultaneous (by the time of formation) (McLaughlin, 1980), acquired only in childhood when the child learns two languages simultaneously from the beginning.

\subsection{Educational and cognitive activity of primary school pupils as an object of study.}

Two types of activities are the key to the full development of the child in primary school. The first type of activity is the learning of the accumulated experience of mankind, and introduction to modern culture. Learning activities are based exactly on this process. The second type is a creative activity that involves an individual self-realization. Unlike the learning one, this type of activity is not aimed at the acquisition of the previously known information, but is focused on the implementation of the learner's own ideas related to the creation of something previously unknown by him.

Learners solve various problems by using the previously mentioned activities. So, educational and training problems are solved during learning activities. Information search problems aimed at the development of the child's abilities are to be solved during the creative activity. If the ability to learn is forming in the process of training activities, the ability to search and find new solutions (on which the bilingual system of intellectual development is focused) is forming in the framework of creative activity.

In today's elementary school, almost during the whole training time, the learners are solving educational, training and routine problems, aimed at a gradual reduction of the children's searching activities with each subsequent task, so that finally in the course of the development of skills in solving problems of a certain type such searching activities will completely disappear. Upon mastering of a method of problem solving, the learner starts using it and not searching for it again and again.

Learners get used to evaluate their own and others' abilities only on the basis of successful or unsuccessful solving of routine problem. And the high self-esteem of a child is related not with a manifestation of his own intelligence, but with due diligence in learning rules and knowledge adoption.

In this connection the important feature of the bilingual system of intellectual development, that allows reconciling educational activity related to the mastering of knowledge and skills in classes with the search, training and creative activities, thereby contributing to the humanization of educational process in elementary school.

\subsection{The technology of implementation of bilingual system of intellectual development.}

Bilingual tasks system is implemented in the form of intellectual training.

The intellectual training is the implementation of a system of creative tasks aimed at the intellectual development of the learner.

The main objectives of the training are:

1) to develop a way of thinking in the process of formation of the basic mental operations: analysis, synthesis, comparison, generalization, classification, ability to allocate the main thing, to prove and disprove and to make conclusions;

2) to develop various types of memory, attention, thinking, perception and imagination;

3) to develop speech skills: ability to prove with reason the own point of view, to make conclusions, to communicate thoughts correctly and clearly;

4) to teach creative thinking skills and to develop ability to solve non-standard problems;

5) to develop the cognitive activity and independent intellectual activity of learners;

6) to develop the ability to communicate and interact with the team members, to work in pairs, groups, to respect the views of others, to objectively evaluate their own work and activities of classmates; 
7) to teach how to apply practically the knowledge and skills obtained during the school subjects study.

The main components of the intellectual training are exercises aimed at the development of the ability to analyze, synthesize and compare; development of the ability to identify cause-and-effect relations and contradictions; to predict the possible course of events; to see all aspects and sides of a situation or object; to create new ideas and present a solution in the form of signs and symbols (tables, diagrams, charts).

Intelligent training requires from learners to perform such types of work as memorization of the information given in different ways, revelation of regularities in the ranks of the signs, selection of words as per a given criterion, manipulation of spatial relations and detailization of visual images.

The tasks, exercises, problems, questions, puzzles and games used in classes are interesting and understandable that is attractive to primary school pupils.

The absence of grades reduces the learners' anxiety and unreasonable uneasiness, the fear of erroneous responses disappears. As a result, the children consider these classes as a means of personal development.

Independent solving by children of search problems takes most of the time in classes. As a result, the children develop the ability to make decisions, to manage themselves in difficult situations and to act independently.

Collective verification of the problem solving is held after the independent work at each lesson. This form of working sets conditions for the normalization of self-esteem in all children, namely: self-esteem rising in children who are well-developed in mental processes, but learn badly the educational material in the classroom due to the lack of attention, for example; other children may start losing their self-esteem because their educational achievements are based mainly on their diligence and studiousness.

During the classes, the joint discussions regarding the way of solving of a certain problem are held. At this stage, the children develop such important qualities as understanding of their own actions, self-control and ability to render an account of the steps followed in solving of a problem of any complexity.

The training contains tasks of varying complexity, and therefore poorly developed children participating in activities feel self-confident.

At these lessons a child assesses himself his successes, which provides a special positive emotional background: relaxedness, interest and desire to learn how to carry out the proposed tasks.

The purposeful development of mental operations starts from the teaching learners to raise questions which stimulate the development of flexibility and comprehensiveness of mental operations as the basis of intellectual abilities.

For each lesson special exercises are selected from the bilingual system as per the level of cognitive development. In order to achieve a developmental effect, tasks of the same type are carried out many times by different ways. One such way is a game.

Gaming technologies are one of unique forms of intellectual development that make the work at creative and exploratory level interesting and fun. In addition to the intellectual games in the form of guessing, pictorial puzzle and problems solving a game design is used.

As a part of the game design the following task are set:

1) tasks focused on the development of non-existent objects, phenomena (fantastic) (for example, a reading task for $2^{\text {nd }}$ grade learners: to prepare a series of illustrations for fairy tales);

2) tasks involving the learners' own observations and experiments (empirical) (for example, an Adyghe language task for $3^{\text {rd }}$ grade learners: to create a collection of riddles);

3) tasks aimed at studying and summarizing of the facts and materials contained in different sources (theoretical) (for example, the mathematics task for $4^{\text {th }}$ grade learners: to create interesting tasks for a quiz).

\subsection{Features of the implementation of the bilingual system of intellectual development.}

In accordance with the characteristics of pupils of primary school age, the scope of the intellectual development of bilingual learners has been defined: educational and play activities.

Assignments are systematized so that one type of activity is replaced by another one, different themes and forms of presentation are actively rotated. This makes the work dynamic, intensive and less fatiguing.

The intellectual potential of the personality of the primary school pupil is disclosed in developing training, if the classes are organized in such a way that learners of different educational success rate are easy carrying out creative tasks, understand that with the help of a certain mental effort they will get the right answer having experienced the joy of learning.

As the results of observations show, in most of the primary school learners the level of intellectual development, related to the ability to generalize, synthesize and integrate the educational material is below the level of development of 
other skills (reading and writing). Therefore, our goal is to pay the greatest attention to the development of such qualities as ability to differentiate, to analyze, to specify (perception of changes after rearrangement and detail addition), to predict (the projection of the consequences) and to maintain accuracy.

In the process of purposeful intellectual development, the learners develop the ability to acquire knowledge by themselves, to formulate and to solve their own problems and to exhibit the personal activity. In the ability to quickly look through many options in order to solve the same problem the level of mental operation speed development is manifested.

In solving of typical problems in various school subjects on the basis of the pre-learned method of their solving, the learners have few opportunities to exhibit in mental activity independence, intelligence and smartness, and for that reason it is important to introduce fragments of intellectual and developmental activities in the primary school education process.

It should be also noted that in the primary school classes the use of regular developing fragments based on materials not related to the education program, eases the tracking of the mental development of each learner beside the educational success that allows timely detection of certain changes in development. Participation of learners in developing lessons fragments (not only educational) makes it possible to obtain information about changes in the selfconsciousness of children, especially in their self-esteem.

During each task almost all cognitive processes are developed, but each time the focus is on one of them. Accordingly, the task system can be presented as subgroups of development-oriented tasks regarding the following: 1) attention, 2) memory, 3) imagination and 4) thinking.

Attention-oriented tasks are focused on the improvement of voluntary attention of children, span of attention, its stability, switching and distributability. Carrying out of tasks from this subgroup promotes the formation and maintenance of the selective focus on the subject and the intensification of cognitive activity focused on the subject.

Memory-oriented tasks are focused on the improvement of auditory and visual memory. Children learn the techniques of memorization. At the same time, the volume of children's visual and auditory memory and replication increases, their logical memory, perception and observation develop and the memorization speed increases.

Imagination-oriented tasks are composed mainly on the material of a geometric nature. This material facilitates the child's passage to a verbal and logical level as it is based on real objects of reality, and corresponds to the primary school pupil's visual-active and visual-imaginative levels of cognitive activity. The system includes such tasks as: to finish a composition of simple geometric bodies or lines; to select the objects of desired shape in order to reconstruct the whole; to draw a unicursal figure (figures, which should be drawn without lifting the pencil from the paper and without drawing the same line twice); to choose a pair of identical objects of complex configuration; to find a disguised drawing in a total drawing composed by given figures; to divide a figure into several given figures and to construct a desirable figure using several parts selected from a great number of them; to compose desired objects using the proposed materials.

The priority direction of the educational process in the primary school is the development of thinking process. In accordance with this purpose, the system includes the thinking-oriented tasks based on materials comprehensible by children. These tasks provide children with possibility, according to their life experience, to find an analogy, to establish associations, to think by them-selves, to be able to see a new side of long familiar and well-known things.

\section{Results}

To keep tracking of the results, the following forms of control have been used:

1) Starting control, helping to determinate the initial level of development of the learners.

2) Routine control: prognostic (trial of all education operations before their actual implementation); functional (control of the accuracy, completeness and consistency of operations included in the educational process); reflexive (control of the possibility of execution of planned actions); control of the result (control carried out after the implementation of actions. It is carried out by comparing actual results or operations performed with the sample).

3) Final control: testing; practical work; creative works of learners.

4) Self-evaluation and self-control (the definition by the pupil of his potential, as well as his understanding of the problems to be solved in the course of carrying out of activities).

In order to identify the level of attention development at the control stage of the experiment the Mustenberg test, the Thorndike test, Ramul Attention Span Test were used (Raigorodskyy, 2005).

The attention of many children of the experimental group (EG) is characterized by arbitrariness. It should be noted that arbitrary attention was developing together with the educational motives, together with an increase in awareness of responsibility for the success of studying. Involuntary attention of many children is particularly concentrated and sustained when the training material is prominent, demonstrative and induces an emotional attitude. The span of attention of EG 
children has increased significantly. Learners stay attentive throughout the lesson that characterizes the stability of their attention. They are able to stay focused on a particular material for a longer period of time. Children can perform several activities at the same time.

In order to identify the level of memory development, the Wechsler intelligence scale and the Benton visual retention test (Raigorodskyy, 2005) were used.

In the EG children the formation of intelligent memorization has been observed. Learners are able to break down the material into semantic units, perform the grouping by meaning, semantic comparison, and as a result they successfully memorize and reproduce the required material. The volume of material memorized by learners has been increased. The strength and accuracy of memory in EG children have been increased. Many children's memory has become more productive.

In order to determine the level of thinking, the "Object classification" and "Object exclusion" (Raigorodskyy, 2005) methods were applied.

At the control stage the primary school pupils' way of thinking is characterized by speed of selection, abstraction and generalization of the essential features of the analyzed situations. Learners are able to deal with the transfer of knowledge in relatively new conditions. Focusing on the analysis of the relationship between the given objects is specific to the learners with a high level of thinking development. EG children express more distinct intelligent mental activity. Children can give a verbal report (adequate in relation to practical actions) on the progress of problem solving and correct their actions. EG learners are able to overcome the barrier of past experience, i.e. the manifestation of an original approach in the analysis of the situation, the restructuring of usual actions, if they cease to meet the requirements of a changing reality; to improve already discovered ways of solving; to shift from direct relationships to the inverse ones. Children have shown intellectual activity, prolonged mental activity, and a great interest in it, its stability and intensity.

The children with low levels of thinking development have expressed the passivity of thinking, stereotypeness, frequent return back to familiar actions even if they are no longer suitable for changed conditions; low mental capacity.

In order to determine the level of imagination development, at the control stage of the experiment we used a "Create a story" method (Raigorodskyy, 2005). The ratio of the results obtained at the determination stage and control stage in order to determine the level of development of the imagination of EG learners has shown a positive dynamics. The observed increase in the control group (CG) is significantly lower than the results obtained in the EG, which confirms the importance and effectiveness of the work conducted at the forming stage of the experiment.

Analysis of the activities of primary school pupils has shown that the children start taking cognitive actions constituting a generalized method of problem solving: theoretical analysis, which allows to allocate the essential relations in problem specification; meaningful reflection as a reference to their own actions related to the understanding of dependence of the way of actions from the essential relations; holistic planning related to the general programming of problem solving.

So, the implementation of bilingual resource in the intellectual development of primary school-aged bilingual learners contributes to their intellectual capacity.

\section{Discussion}

Comparison and complex analysis of the results of the bilingual learners' intellectual development diagnostic, and comparison of the data with the results obtained at the determination stage give the possibility to state the following: at the end of the experiment, the great majority among the learners with a high level of intellectual development were those who have implemented the bilingual system of intellectual development.

The implementation in the educational process of bilingual intellectual development system enhances the development of educational and intellectual abilities of primary school pupils.

Essential capacities of the learner acquire the power, strength, depth and flexibility because of the activation of different types of thinking. There is an enrichment of the learner's vocabulary, development of his cognitive activity and the knowledge interchange.

The intellectual potential of learners is enhanced due to the complex development of different types of memory, attention, observation, imagination, perception, as well as due to the formation of unconventional thinking and language development. The use by the teacher in the educational process of special elaborated intellectual training tasks, with gradually increasing complexity from lesson to lesson and from class to class facilitates the sustained intellectual development of learners.

When analyzing the results by the end of the experiment, the following patterns of intellectual development of bilinguals were confirmed empirically: 
1. Intellectual development of bilinguals directly depends on the actualization of both linguistic structures (mother and non-mother tongues) in the educational process.

2. Maturity of the experience of mental activity in two languages (mother and non-mother tongues) ensures the effectiveness of the intellectual development of bilingual learners.

3. Activation of linguistic structures of the person (mother and non-mother tongues) in the cognitive tasks solving facilitates the development of bilingual experience in learners' mental activity.

\section{Conclusions}

The study allowed us to make the following conclusions:

1. The strategic resource of bilingualism is based on the following complex: knowledge of languages (mother and non-mother tongues), each of which is a reflection of the culture, worldview and attitudes of different ethnic groups; the experience of mental activity on the basis of learners' language structures; experience of motivational and value attitude towards the society, pupils themselves, knowledge, skills, which is a framework for the development of mental abilities of pupils; the complex of knowledge and skills that reflects the level of development of mental abilities.

2. Bilingualism is a resource of learners' intellectual development. The objective part of the strategic resource of bilingualism is the presence of a double sign and symbolic systems, i.e. two means of thought formation. The subjective part is revealed in distinguishing characteristics of bilinguals: a creative approach to the handling of information of different levels, including sensitivity of the bilingual to semantic relationships between the words; the ability to generalize the conclusions in two languages; the ability to perform the tasks related to the verbal transformation and replacement; a developed linguistic memory; linguistic competence.

3. The implementation of bilingual resource in the educational process increases the intellectual potential of primary school pupils. Gradual systematic intellectual development with the use of tasks system based on symbolic codes of the two languages (mother and non-mother tongues), non-related to the educational material, facilitates the qualitative and quantitative intelligent development of bilingual learners.

The prospects for further study of the problem of mental abilities of bilingual learners, in our opinion, are:

- Development of mental abilities of bilingual learners in the environment of the Russian national bilingualism (as exemplified by different national republics);

- Development of a bilingual system of exercises of mental abilities of bilingual learners based on the material of mid-level school subjects (senior school);

- Development of conceptual provisions for specialist training (primary school teachers, educational psychologists) for the work in educational institutions under conditions of the Russian national bilingualism.

\section{Acknowledgment}

We express our gratitude to the Adyghe State University administration for the financial support in realization of this publication.

\section{References}

Avrorin, V. A. (1975). Problems of studying the functional part of the language (To the question about the subject of sociolinguistics). Leningrad: Nauka.

Bogoyvlenski, A. N. (1992). Intellectual activity as the problem of creativity. - Moscow: Education.

Bogus, M. B. (2008) Effect of bilingualism on the intellectual development of the individual trainees. Bulletin of the Adyghe state University. Ser. Pedagogy and psychology.7 (pp. 47-52).

Davydov, V. C. (1982). The Content and structure of educational activity of learners . Formation of educational activity of learners (pp. 6578).

Davydov, V. V. (1986). Problems of developmental education: the Experience of theoretical and experimental research. - Moscow: Education.

Dyachkov, M. C. (1991). Problems of bilingualism (multilingualism) and education. - Moscow: In-t NAT. problems of the municipality of the RSFSR.

Elkonin, D. B. (1961).Psychology of teaching primary school children. Retrieved from [http://psychlib.ru/mgppu/EPr-1997/EPR-2391.htm.

Kalmykov, H. I. (1981). Productive thinking as the basis of learning. - Moscow: Education.

Matyushkin, A. M. (2008). Problem situations in thinking and learning. - Moscow: Direct Media Publishing. 
Melnikov, A. A. (2003). Language and national character. The correlation between the structure of language and mentality. St.Petersburg: Speech.

Menchinscay, N. A. (1989).The learning problems and mental development of the learner. - Moscow: Education.

Mikhailov, M. M. (1989). Bilingualism: problems, searches... - Cheboksary: Chuvash. kN. Publishing house.

Osgood, C. E. (1980). Lectures on language performance. - New York: Springer.

Psycholinguistic studies of speech development and second language learning. (Coll. of art.) (1980). - Moscow: The Labyrinth.

Raigorodskyy, D.Ya (2005). (Ed.) Practical diagnostics. Methods and tests: textbook. - Samara: Bahram-M.

Repkin,V.V. (1976). Centuries the Structure of learning activities. Journal of Kharkiv University. 132. (pp. 10-16).

Sukhomlinsky, B. A. (1983). Education About mental. - Kiev: visa SHK.

Vergeles, G. I. (1990). Didactic bases of formation of educational activity primary school. -Leningrad.

Vereshchagin, E. M. (1969). Psychological and methodological characteristics of bilingualism (bilingual). Moscow: Moscow State University Press.

Vygotsky, L. S. (1982). Thought and speech. - Moscow: Education.

Zack, A. Z. (2004).Thinking primary school children: study, diagnosis, formation. - St. Petersburg: Assistance.

Zhoy, K. K. (1990). Language as practical consciousness. - Kiev: Visa SHK. 